Check for updates

Cite this: RSC Adv., 2018, 8, 31979

\title{
One-pot synthesis of cup-like ZSM-5 zeolite and its excellent oxidative desulfurization performance $\uparrow$
}

\author{
Yuan Zhang, ${ }^{a}$ Wei Zhang, ${ }^{\text {*a }} \mathrm{Ju}_{\text {Zhang, }}{ }^{\mathrm{a}}$ Zhao Dong, ${ }^{\mathrm{a}}$ Xiongfu Zhang (D) \\ and Shujiang Ding (iD *c
}

A one-step method to synthesize small size cup-like hollow ZSM-5 single crystals is reported in this paper. Characterization methods show that the product has a typical ZSM-5 structure and most crystals are uniform, mono dispersed crystal shells with distinct hollow structure. The average size of a single crystal is about $350 \mathrm{~nm}$. Every cup-like hollow ZSM-5 crystal can act as a microreactor in the reaction, and the ZSM-5 based catalyst POM-MOF-ZSM-5 (polyoxometalates (POM), metal-organic frameworks (MOF)) shows excellent oxidative desulfurization performance of DBT (dibenzothiophene).

Received 21st August 2018

Accepted 7th September 2018

DOI: $10.1039 / \mathrm{c} 8 \mathrm{ra0} 0984 \mathrm{k}$

rsc.li/rsc-advances

Currently, there are only limited reports on hollow, single

\section{Introduction}

Zeolites are widely used in industry as heterogeneous catalysts and especially in oil refining and petrochemistry as solid acid catalysts. ${ }^{1}$ However, zeolites have diffusional limitations which bring lots of problems such as decrease in reaction rate and deactivation. ${ }^{2,3}$ Decreasing the diffusion length in the zeolite crystals is a way to alleviate molecular transport problems. But their synthesis is not always easy. Therefore, to create an additional array in zeolites, fabrication of zeolite molecular sieves with mono-crystalline hollow structures attracts wide attention. ${ }^{4,5}$ In hollow structure zeolites, guest molecules only stay in the zeolites for a short amount of time, thus improving the mass transfer efficiency. ${ }^{6-9}$ In addition, small size crystal molecular sieves have larger specific surface area, shorter pore channels, abundant surface acid sites compared to big size crystal molecular sieves. ${ }^{7}$ Therefore, small size crystal molecular sieves are more stable, more active and more selective, and they have shown excellent catalytic performance in many critical catalytic reactions..$^{9,10}$ Besides, the large internal cavity of hollow structure zeolites can encapsulate catalyst and be used as a micro reactor. Compared to polycrystalline hollow zeolites, hollow single crystals are generally defect-free and homogeneous, which make it perfectly adapted for the encapsulation of catalysts. ${ }^{11}$ They also have the potential application as biomedical and pharmaceutical vectors for controlled drug delivery.

${ }^{a}$ Research Institute of Yanchang Petroleum (Group) Co., LTD., Xi'an 710075, China. E-mail: ycsy_zw@163.com

${ }^{b}$ National Key Laboratory of Fine Chemicals, School of Chemical Engineering, Dalian University of Technology, Dalian, 116024, China

'Department of Applied Chemistry, School of Science, Xi'an Jiaotong University, Xi'an, 710049, China.E-mail: dingsj@xjtu.edu.cn

$\dagger$ Electronic supplementary information (ESI) available. See DOI: 10.1039/c8ra06984k crystal ZSM-5 zeolite molecular sieves. These reports are mainly focused on MFI molecular sieves ${ }^{12-17}$ due to their excellent pore channel structure, thermo stability and thus vast potential in many catalytic reactions.

One method of synthesizing hollow ZSM-5 crystals includes two steps. Firstly, they manufactured conventional ZSM-5 molecular sieves grain crystal, and then treated the solid crystals with acidic or basic solutions for dealumination or desilication. Finally, they did recrystallization to obtain some hollow zeolite molecular sieves. ${ }^{12-19}$ Mei and colleagues $^{20}$ treated $350 \mathrm{~nm}$ ZSM-5 molecular sieves with $0.6 \mathrm{M} \mathrm{Na}_{2} \mathrm{CO}_{3}$ to obtain small-sized hollow ZSM-5 crystal. Fodor et al. ${ }^{21}$ first synthesized nanoscale ZSM-5 molecular sieves, and obtained nanoscale hollow ZSM-5 molecular sieves by first corroding with $0.1 \mathrm{M}$ sodium hydroxide and then washed with $0.1 \mathrm{M}$ hydrochloric acid.

Another method to synthesize hollow ZSM-5 crystals is the addition of the hard templates or soft templates. During the synthesis, carbon nanoparticles or some polymer beads was used as hard templates. ${ }^{22-24}$ Jacobsen et al. ${ }^{25}$ used excess of a zeolite gel to grow around carbon particles of the inert matrix within the whole pore system. After the combustion in air, the carbon particles were removed and the mesoporous zeolite single crystals were obtained. Zhao et al. ${ }^{26}$ reported the fabrication of mesoporous zeolite with a hollow capsule structure by using conventional TPAOH and surfactant (CTAB) as soft microand mesopore generation templates through controlled hydrolysis.

The aforementioned reports regarding synthesizing hollow zeolite molecular sieves all have relatively complex synthesis process, and need further treatment to obtain somewhat hollow molecular sieves. These process is time and energy consuming, and potentially harmful for the environment. Therefore, seeking a simple, single-step method for synthesizing small 
crystal hollow ZSM-5 zeolite molecular sieves remains a challenge.

This article introduces a one-step, simple method for directly synthesizing hollow, small-sized single crystal ZSM-5 zeolite. It has cup-like structure and the uniform shell is dozens of nanometers. The cavity can encapsulate catalyst, and act as a microreactor. Therefore, the catalyst POM-MOF-ZSM-5 was prepared, which shows excellent oxidative desulfurization performance of DBT and huge potential in the desulfurization of the oil.

\section{Results and discussion}

\subsection{Synthesis and characterization}

We mixed sodium metaaluminate, tetraethoxysilane (TEOS), tetrapropylammonium hydroxide (TPAOH) and water in a certain order until the mixture is uniform. The mixture was put in crystallization reactor and crystallized under certain temperature for 12 to 48 hours. Then the products was filtered, washed, and dried to obtain small-sized hollow ZSM-5 molecular sieves. Finally, the sample was converted into H-ZSM-5 through ion exchanges with $\mathrm{NH}_{4} \mathrm{NO}_{3}$ solution at $90{ }^{\circ} \mathrm{C}$ for three times and calcinated at $550{ }^{\circ} \mathrm{C}$ for $5 \mathrm{~h}$.

Both XRD and IR results show that the synthesized hollow molecular sieves (crystallization temperature $130{ }^{\circ} \mathrm{C}$, crystallization time $48 \mathrm{~h}, \mathrm{OH}^{-} / \mathrm{SiO}_{2}=0.3$ ) have the framework structure of ZSM-5 (Fig. 1). The overall graphs exhibit no impure crystal peaks and no incompletely crystallized amorphous plastic materials. The three $\mathrm{NH}_{3}$ desorption peaks of the $\mathrm{NH}_{3}$-TPD infers the existence of the three kinds of acids, weak acid, mediate strong acid and strong acid (Fig. S1 $\dagger$ ). The XPS of the catalyst gives the information about the chemical state (Fig. S2 $\dagger$ ). The binding energy peaks of $\mathrm{C}, \mathrm{O}, \mathrm{Si}, \mathrm{Al}$ are easy to be confirmed in the survey spectrum. The nitrogen adsorptiondesorption isotherms of the cup-like ZSM-5 zeolite exhibits the type II isotherms and proves the existence of the large pores (Fig. S3†).

Nextly, we evaluated the SEM and TEM results of the synthesized hollow ZSM-5 (Fig. 2b and d) comparing to regular, solid ZSM-5 sample (Fig. 2a and c). From SEM (Fig. 2b), it is seen that the crystals are hollow and the size of zeolite particle is about $350 \mathrm{~nm}$. From TEM, ZSM-5 sample in Fig. 2c are opaque and non-transparent; whereas the crystals in Fig. $2 \mathrm{~d}$ are transparent and thus show typical hollow cup-like structure, whose shell thickness is dozens of nanometers. Therefore, this method


Fig. 1 XRD and IR of regular solid and hollow ZSM-5 molecular sieves (a) XRD. (b) IR

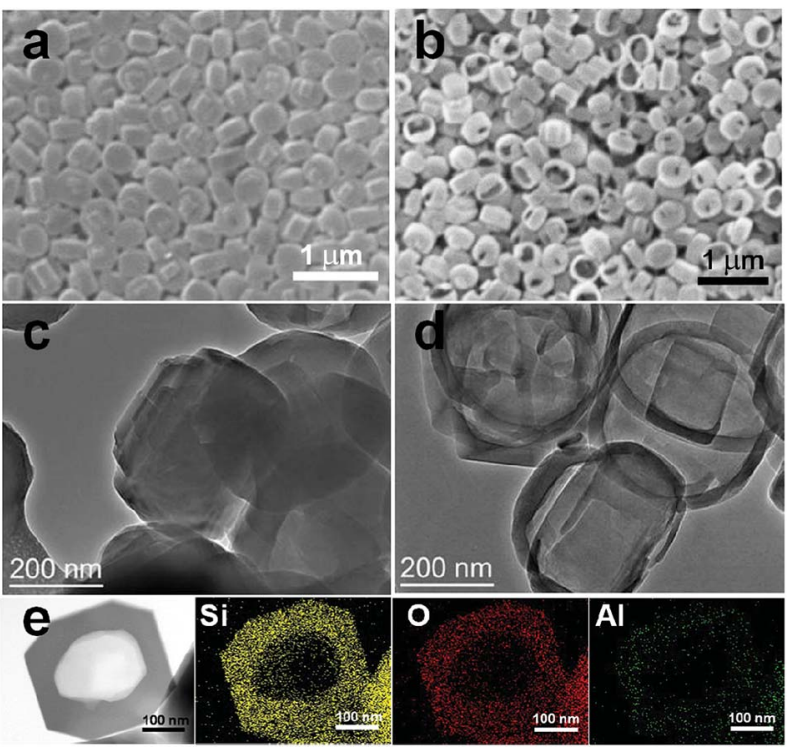

Fig. 2 SEM ( $a$ and $b$ ) and TEM (c and d) of synthesized zeolites. ( $a$ and c) Regular, solid ZSM-5. (b and d) Hollow ZSM-5. (e) Elemental mapping images of an individual ZSM-5 crystal.

of synthesis yields hollow zeolite single crystals. In addition, the resulted molecular sieves have uniform shell thickness and well ordered pore channel structure. From elemental mapping images of an individual ZSM-5 crystal (Fig. 2e), the shell of the ZSM-5 crystal is mainly formed by silicon and oxygen, together with a little amount of aluminum. It is believed that the cup-like structure can be used as a microreactor in reactions.

\subsection{Key influence factors in synthesizing hollow ZSM-5 zeolites}

2.2.1 The effect of crystallization time. Fig. 3 is the SEM image of ZSM-5 molecular sieves samplent crystallization time, while holding all other parameters constant. Clearly, crystallization time has critical influence on forming ZSM-5 molecular sieves. When crystallized for $12 \mathrm{~h}$ or $24 \mathrm{~h}$, the resulted product

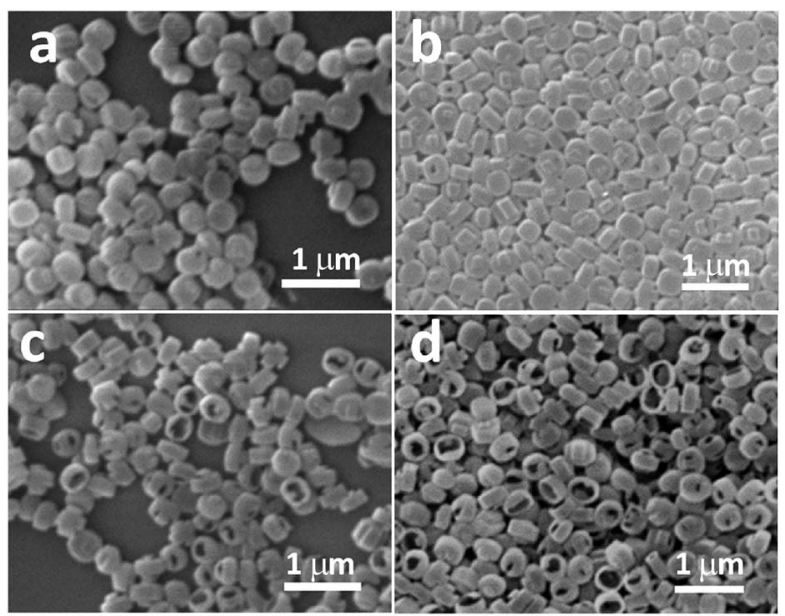

Fig. 3 SEM of synthesized ZSM-5 with different crystallization time $\left(130{ }^{\circ} \mathrm{C}_{1} \mathrm{OH}^{-} / \mathrm{SiO}_{2}=0.3\right)$. (a) $12 \mathrm{~h}$, (b) $24 \mathrm{~h}$, (c) $36 \mathrm{~h}$, (d) $48 \mathrm{~h}$. 


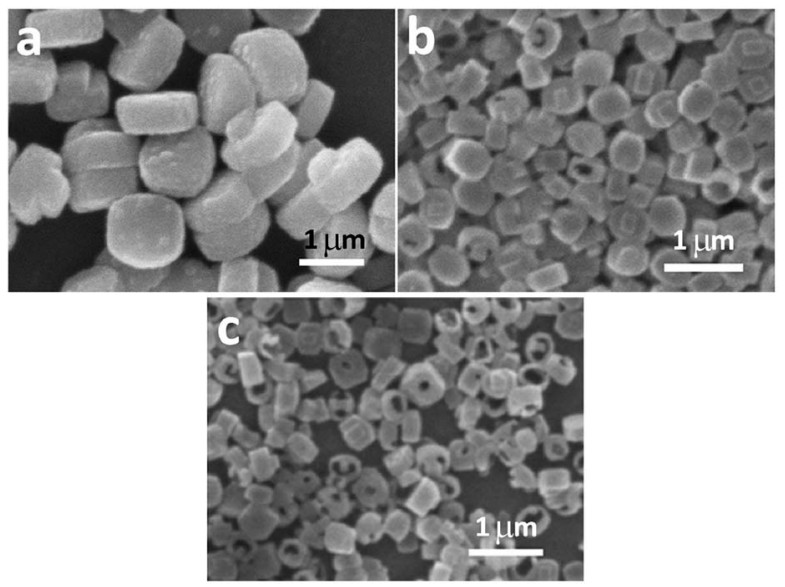

Fig. 4 SEM of ZSM-5 with different basicity while crystallized for $48 \mathrm{~h}$ under $170{ }^{\circ} \mathrm{C}$. (a) $\mathrm{OH}^{-} / \mathrm{SiO}_{2}=0.17$; (b) $\mathrm{OH}^{-} / \mathrm{SiO}_{2}=0.3$; (c) $\mathrm{OH}^{-} / \mathrm{SiO}_{2}=$ 0.4 .

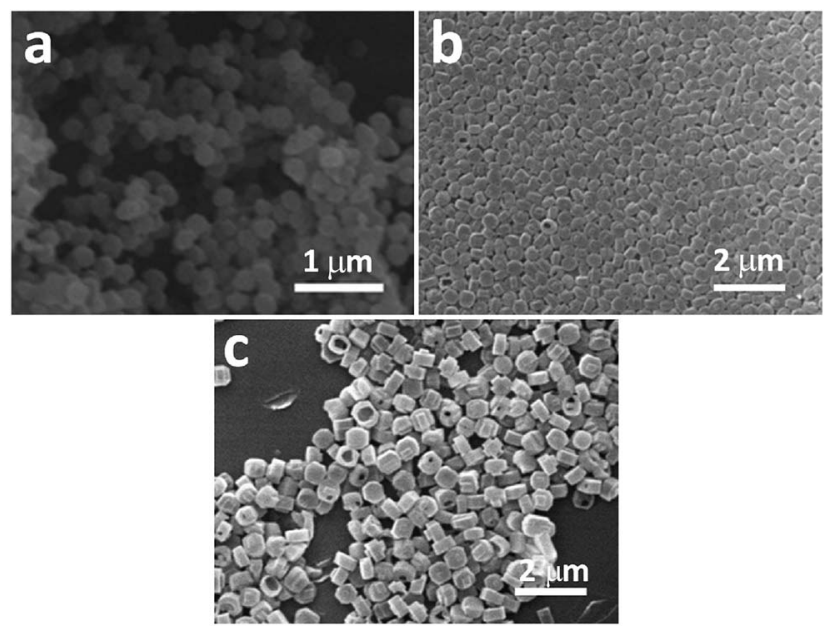

Fig. 5 SEM of ZSM-5 under different crystallization temperature $\left(\mathrm{OH}^{-} / \mathrm{SiO}_{2}=0.3\right.$, crystallized for $\left.24 \mathrm{~h}\right)$. (a) $100^{\circ} \mathrm{C}$, (b) $130{ }^{\circ} \mathrm{C}$, (c) $170^{\circ} \mathrm{C}$.

was solid ZSM-5 crystal. When the crystallization time prolonged to 36 hours, some of the synthesized ZSM-5 particles became hollow. When it was crystallized for 48 hours, the resulted crystals were mostly hollow ZSM-5. Therefore, it is concluded that long crystallization time is beneficial for forming hollow ZSM-5. We further deduce that the synthesis process involves first forming solid ZSM-5 crystals, then converting to hollow crystals under the corrosion of bases present in the system.

2.2.2 The effect of synthetic basicity. Fig. 4 is the SEM image of synthesized ZSM-5 with different synthetic fluid basicity. The picture shows that when the synthetic system was less basic $\left(\mathrm{OH}^{-} / \mathrm{SiO}_{2}=0.17\right)$, the synthesized molecular sieves were solid ZSM-5 particles with relatively big particle diameters. When the basicity increased $\left(\mathrm{OH}^{-} / \mathrm{SiO}_{2}=0.4\right)$, the resulted zeolite crystals were mostly hollow ZSM-5 with uniform particle diameters and similar shapes. Therefore, high basicity is a favorable condition for forming hollow ZSM-5 crystals.
2.2.3 The effect of crystallization temperature. The SEM image of synthesized ZSM-5 with different crystallization temperature is shown in Fig. 5. There was no hollow ZAM-5 crystals when the crystallization time was $100{ }^{\circ} \mathrm{C}$. And there was very little hollow ZSM-5 among the solid ones crystallized at $130{ }^{\circ} \mathrm{C}$. Whereas at $170^{\circ} \mathrm{C}$, the synthesized ZSM- 5 crystals were mostly hollow. Therefore, high crystallization temperature is favorable for hollow ZSM-5 formation.

\subsection{Discussion on the mechanism of hollow ZSM-5 formation}

As discussed in the previous section, long crystallization time, high crystallization temperature and high system basicity are all favorable conditions for forming hollow ZSM- 5 crystals. Therefore, we deduce that there are two steps in this synthetic process: first the crystallization of regular solid ZSM- 5 crystals; then the corrosion of these solid crystals under basic conditions. As the crystallization time increases, the crystal growth slows down and the corrosion speeds up.

This deduced mechanism is consistent with our observations. When crystallized for only 24 hours, the ZSM-5 crystals were mostly solid. It is suggested that the early synthetic environment favours the formation of zeolite crystals and the corrosion is weak. When crystallized for 36 hours and even 48 hours, it was observed that the particle diameter of zeolite crystals remained constant, while more and more crystals became hollow. It is indicated that after 24 hours, the synthetic environment does not support crystal growth but favours corroding zeolite crystals.

When crystallized for a longer time (48 hours) under low system basicity (Fig. 4a), the zeolite crystals were mostly solid and the crystals have clearly grown to about $1 \mu \mathrm{m}$. It is indicated that low system basicity is beneficial for zeolite crystal growth, not basic corrosion of crystals.

This one-step synthetic method for single crystal hollow ZSM-5 zeolites has similar mechanism as the previously reported two-step methods. ${ }^{6,8,10}$ The key in the single-step synthesis is the coordination between different parameters. In the same synthetic system, the zeolite crystals grow in the earlier stage. Afterwards the crystals are corroded to form molecular sieves with hollow structures.

\subsection{Oxidative desulfurization performance of POM-MOF- ZSM-5}

In earlier report, a catalyst system was designed by a kind of hybrid material POM@MOF-199@MCM-41 (PMM). It was fabricated through a one pot and POM template selfconstruction of MOF-199 in the pore of MCM-41. ${ }^{27}$ This catalyst was used for the direct oxidative desulfurization process of model oil. In our work, we used hollow ZSM-5 instead of MCM41 to obtain the catalyst, which gave excellent desulfurization performance.

ZSM-5 was added into polyoxometalates (POMs) $\mathrm{PMo}_{12} \mathrm{O}_{40^{-}}$ $\cdot n \mathrm{H}_{2} \mathrm{O}$ solution, then metal-organic frameworks (MOFs) MOF199 was added in. The mixture was refluxed overnight, filtered 
Table 1 Desulfurization performance of different catalysts

\begin{tabular}{ll}
\hline Catalyst & DBT conversion (\%) \\
\hline ZSM-5 & $21.2 \%$ \\
POM-MOF-MCM-41 & $83.5 \%$ \\
POM-MOF-ZSM-5 & $91.2 \%$ \\
\hline
\end{tabular}

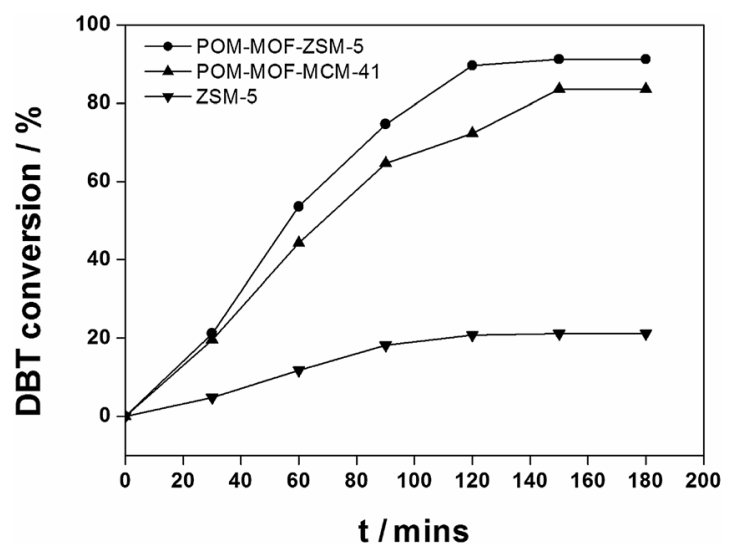

Fig. 6 The desulfurization result of ZSM-5, POM-MOF-MCM-41 and POM-MOF-ZSM-5.

and dried to obtain the catalyst POM-MOF-ZSM-5. We prepared POM-MOF-MCM-41 in the similar way.

DBT was dissolved in octane to obtain model diesel. The oxidative desulfurization experiment was carried out in a $100 \mathrm{~mL}$ three-neck flask connected with a pump to share oxygen. In every run, a certain amount of catalyst was added and stirred. Then $1 \mathrm{~mL}$ reactant solution was collected and centrifuged, and injected in gas chromatograph to calculate the desulfurization rate, following the equation below $(\eta$ is the conversion rate, and $C_{0}$ and $C$ is the initial and final concentration of S-compounds respectively):

$$
\eta=\left[\left(C_{0}-C\right) / C_{0}\right] \times 100 \%
$$

Desulfurization performances of different catalysts were shown in Table 1. The desulfurization rate of ZSM-5 and POMMOF-MCM-41 was only $21.2 \%, 83.5 \%$ respectively, while the value of POM-MOF-ZSM-5 reached 91.2\%. In Fig. 6, after $180 \mathrm{~min}$, the oxidative desulfurization rate increased in the order of ZSM-5 < POM-MOF-MCM-41 < POM-MOF-ZSM-5. The reactivity of POM-MOF-ZSM-5 is the best, for the more firmly and uniformly fixation of heteropolyacid in MOF-199, after dispersed homogeneously in the hollow ZSM-5 zeolites. More detailed results will be reported later.

\section{Conclusions}

This single-step, hydrothermal synthesis method yielded highly crystallized small size cup-like ZSM-5 crystal with uniform shell thickness and well-ordered pore channels. The resulted zeolite crystals are mono dispersed, highly crystallized. These zeolite crystals have uniform particle diameters (approximately 350 $\mathrm{nm}$ ) and no impure crystals. Long crystallization time, high crystallization temperature and high synthetic basicity are favorable conditions for forming hollow ZSM-5 molecular sieves. The synthetic process for hollow ZSM-5 molecular sieves have simple operation steps without any additives, and can yield high quality molecular sieve products. The whole process is fast, energy saving and environment protecting. The catalyst POM-MOF-ZSM-5 based on hollow ZSM-5 molecular sieves exhibits good oxidative desulfurization performance of DBT, which implies a broad application prospect.

\section{Conflicts of interest}

There are no conflicts to declare.

\section{Acknowledgements}

This work was supported by Foundation of Yanchang Petroleum (Group) Co. (No. ycsy2015ky-A-12 and ycsy2017QNJJ-b-19).

\section{Notes and references}

1 M. E. Davis, Ind. Eng. Chem. Res., 1991, 30, 1675.

2 C. Yu, J. Fan, B. Tian, et al., Adv. Mater., 2002, 14, 1742.

3 M. Hartmann, Angew. Chem., Int. Ed., 2004, 43, 5880.

4 F. Caruso, R. A. Caruso and H. Moöhwald, Science, 1998, 282, 1111.

5 X. Wang, X. Zhang, Y. Wang, et al., Chem. Mater., 2011, 23, 4469.

6 S. Van Donk, A. H. Janssen, J. H. Bitter, et al., Catal. Rev., 2003, 45, 297.

7 R. Ravishankar, C. E. A. Kirschhock, P. P. Knops-Gerrits, et al., J. Phys. Chem. B, 1999, 103, 4960.

8 W. Song, R. E. Justice, C. A. Jones, et al., Langmuir, 2004, 20, 8301.

9 W. Zhang, D. Ma, X. Liu, et al., Chem. Commun., 1999, 1091.

10 Y. Wang, M. Lin and A. Tuel, Microporous Mesoporous Mater., 2007, 102, 80.

11 C. Pagis, A. R. Morgado Prates, D. Farrusseng, et al., Chem. Mater., 2016, 28, 5205.

12 W. Song, C. Dai, Y. He, et al., Appl. Catal., A, 2012, 453, 272. 13 V. Valtchev, Chem. Mater., 2002, 14, 4371.

14 Y. Wang and A. Tuel, Microporous Mesoporous Mater., 2007, 113, 286.

15 C. Dai, A. Zhang, L. Li, et al., Chem. Mater., 2013, 25, 4197.

16 J. C. Groen, J. C. Jansen, J. A. Moulijn, et al., J. Phys. Chem. B, 2004, 108, 13062.

17 C. Mei, Z. Liu, P. Wen, et al., J. Mater. Chem., 2008, 18, 3496. 18 J. C. Groen, T. Bach, U. Ziese, et al., J. Am. Chem. Soc., 2005, 127, 10792.

19 Y. Wang and A. Tuel, Microporous Mesoporous Mater., 2008, 113, 286.

20 C. Mei, Z. Liu, P. Wen, et al., J. Mater. Chem., 2008, 18, 3496.

21 D. Fodor, L. Pacosová, F. Krumeich, et al., Chem. Commun., 2014, 50, 76. 
22 A. Zhang, Y. Zhang, N. Xing, et al., Chem. Mater., 2009, 21, 25 C. J. H. Jacobsen, C. Madsen, J. Houzvicka, et al., J. Am. 4122. Chem. Soc., 2000, 122, 7116.

23 J. Liu, S. Z. Qiao, S. Budi Hartono, et al., Angew. Chem., Int. 26 J. Zhao, Z. Hua, Z. Liu, et al., Chem. Commun., $2009,7578$. Ed., 2010, 122, 5101.

27 S. W. Li, R. M. Gao, R. L. Zhang, et al., Fuel, 2016, 184, 18.

24 K. Kamata, Y. Lu and Y. Xia, J. Am. Chem. Soc., 2003, 125, 2384. 